\title{
A TESE DA VERACIDADE NA TEORIA DA INFORMAÇÃO FORTEMENTE SEMÂNTICA DE FLORIDI E O PARADOXO DE BAR-HILLEL-CARNAP
}

\section{THE VERACITY THESIS IN FLORIDI'S THEORY OF STRONGLY SEMANTIC INFORMATION AND THE BAR-HILLEL-CARNAP PARADOX}

Bernardo Gonçalves Alonso*

RESUMO - Neste artigo defendo que a Teoria da Informação Fortemente Semântica de Floridi (2004) - TIFS - está correta ao assumir a Tese da Veracidade, que por sua vez orienta a definição de informação semântica como " $p$ é informação se e somente se $p$ é constituído por dados bemformados, com significado e verdadeiros". Argumento que a teoria não é arbitrária, pois dá conta do desembaraço de conundrums filosóficos importantes, principalmente por evitar o paradoxo de Bar-Hillel e Carnap (1953), que é gerado a partir da teoria clássica da informação semântica. Primeiro é discutido um dos principais resultados da teoria clássica, o de produzir "sentenças muito informativas para serem verdadeiras". Depois são resumidas as motivações para a elaboração de uma "lógica de estar informado" e é mostrado como o sistema KTB-IL é montado e modelado mantendo-se entre os seus axiomas o da veracidade $-\mathrm{K}$ ou $A_{4}$. Finalmente, a TIFS é examinada e defendida ao mostrar que ela restringe aleticamente a extensão do conceito clássico de informação para evitar problemas com tautologias e contradições. A TIFS oferece uma solução original ao capturar nossas intuições modais a respeito da informatividade como noção básica.

PALAVRAS-CHAVE - Lógica de estar informado. Paradoxo de Bar-Hillel e Carnap. Teoria da informação fortemente semântica. Tese da veracidade.

ABSTRACT - In this article I defend that Floridi's Theory of Strongly Semantic Information - TSSI - is correct while encompassing the Veracity Thesis, which guides the semantic information definition as

* Doutor em Filosofia pela UFRJ/University of Hertfordshire. Professor Adjunto da UFMT.

\begin{tabular}{|l|l|l|l|l|l|}
\hline Veritas & Porto Alegre & v. 57 & n. 2 & maio/ago. 2012 & p. 123-142 \\
\hline
\end{tabular}


" $p$ is information if and only if $p$ is constituted by meaningful, truth well-formed data". I argue that the theory is not arbitrary because it deals with important philosophical conundrums, mainly by avoiding the Bar-Hillel and Carnap paradox (1953) generated from the classical theory of semantic information. First, one of the classic theory's main result is discussed: the production of "too much informative sentences to be true". Then the motivations to elaborate a "logic of being informed" are summarized and it is shown how the KTB-IL system is built and modelled keeping the veracity axiom among its axioms $-\mathrm{K}$ or $A_{4}$. Finally the TSSI is examined and defended by showing that it aletically restricts the extension of the classic concept of information, avoiding problems with tautologies and contradictions. The TSSI offers an original solution by capturing our modal intuitions concerning informativeness as a basic notion.

KEYWORDS - Bar-Hillel-Carnap paradox. Logic of being informed. Theory of strongly semantic information. Veracity thesis.

\section{Introdução}

Não existe uma definição unificada sobre o que significa "informação". Por ser uma noção tão amplamente usada em praticamente todas as áreas do conhecimento, "informação" é um conceito polissemântico que pode ser associado a várias explicações, com os mais diversos propósitos. Um importante teórico da informação que compreendeu isso cedo foi Claude E. Shannon ${ }^{1}$ :

"The word 'information' has been given different meanings by various writers in the general field of information theory. It is likely that at least a number of these will prove sufficiently useful in certain applications to deserve further study and permanent recognition. It is hardly to be expected that a single concept of information would satisfactorily account for the numerous possible applications of this general field" (Shannon 1993, p. 180).

Afinal de contas, filósofos não são experts em definir qual o significado preciso de cada palavra? Se usamos indiscriminadamente o termo "informação", faz-se oportuno apresentar a Teoria da Informação Fortemente Semântica de Luciano Floridi (2004) e tentar inoculá-la de algumas críticas para que, no final, tenhamos uma boa noção a respeito do que estamos falando. Tentaremos avaliar se a teoria da informação de Floridi tem alguma utilidade teórica ao mostrar que não é arbitrária,

1 Gostaria de agradecer os comentários, críticas e sugestões dos professores e colegas Luciano Floridi, Jean-Yves Béziau e Andre Furhmann e à agência de fomento CAPES por financiar o meu estágio de doutorado na Inglaterra. 
pois dá conta do desembaraço de conundrums filosóficos importantes, principalmente por evitar o paradoxo de Bar-Hillel e Carnap (1953), que é gerado a partir da teoria clássica da informação semântica, também conhecida como Teoria da Informação Semântica Fraca (Floridi, 2004).

Só que o fato da teoria forte de Floridi evitar o paradoxo de Bar-Hillel e Carnap - e de fato evita, como será mostrado - não é suficiente para estabelecer que a sua noção de informação semântica não é arbitrária (Crnkovic, 2005). E isso se dá porque uma teoria sempre pode evitar determinados resultados indesejados ao afastá-los acrescentando ou eliminado determinados axiomas (Fuhrmann, 1989; Girle, 2000; Floridi, 2006). Neste artigo, tentaremos mostrar que a teoria da informação fortemente semântica de Floridi, além de evitar o paradoxo, não é arbitrária, pois oferece uma solução original ao capturar nossas intuições modais mais profundas a respeito da informatividade como noção básica.

\section{Teoria Clássica da Informação}

A teoria da informação semântica desenvolvida por Bar-Hillel e Carnap (1953) foi construída utilizando-se o aparato do espaço modal de Carnap (1947), que foi usado para definir sua noção de "intensão" e que serve à explicação de necessidade metafísica. Para o nosso propósito, vamos ignorar alguns dos detalhes do trabalho de Carnap e da complexa teoria de Floridi - o presente trabalho não faz uso de nada mais rebuscado do que o cálculo proposicional e recursos simples de lógica modal normal -, no entanto, faz-se oportuno comentar alguns detalhes fundamentais.

A chamada teoria clássica (ou fraca) da informação semântica é construída em torno de uma linguagem predicativa monádica. O número de mundos possíveis é calculado da seguinte maneira. Se temos $n$ constantes individuais (para $n$ indivíduos) e $m$ predicados monádicos primitivos, o número de sentenças atômicas será $\mathrm{nm}$, o número de mundos possíveis $2 n m$. O número de predicadores- $Q$ será $2 m$ - predicadores- $Q$ são individuações de tipos possíveis de objetos dada a conjunção de predicados por meio dos quais cada predicado primitivo ocorre. Uma sentença completa de um predicador- $Q$ é uma sentença $Q$, aonde um predicado é ligado a um termo. Assim, um mundo possível é uma conjunção de $n Q$ sentenças, de modo que cada sentença- $Q$ descreve um indivíduo possivelmente existente (Carnap, 1947).

A intensão de uma sentença declarativa é tida como o conjunto de mundos possíveis que fazem a sentença verdadeira (são os mundos incluídos pela sentença). A informação semântica também é referida por Bar-Hillel e Carnap (1953) como "conteúdo" (cont.) e é tida como o conjunto de mundos possíveis que fazem a sentença falsa (os mundos 
excluídos pela sentença). O conteúdo de uma sentença declarativa é interdefinível com a noção de intensão.

De modo resumido:

- Intensão = Proposição = mundos incluídos pela sentença;

- Conteúdo = Informação semântica = mundos excluídos pela sentença.

Se tomamos $W$ como sendo o conjunto de todos os mundos possíveis, $X$ o conjunto de mundos possíveis identificados com a intensão de uma sentença declarativa $s$, e $Y$ o conjunto de mundos possíveis identificado com o conteúdo de $s$, temos:

$$
W \mid X=Y \text { sse } W \mid Y=X
$$

Ou seja, $X$ e $Y$ sempre são mutuamente excluídos e conjuntamente exauridos em $W$, pois cada um é uma partição de $W$. Dito de outra maneira, o conteúdo de $s$ é idêntico ao conjunto de mundos possíveis que são incluídos pela negação de $s$, quer dizer, o conteúdo de s é identificado com o conjunto de mundos incluídos por $\neg s$, temos que:

- definição [1]: Cont $(s)={ }_{d f}\{x \rightarrow W: x \rightarrow \neg s\}$

Para qualquer sentença logicamente verdadeira $T, \neg T$ vai excluir todos os mundos possíveis:

$$
\operatorname{Cont}(T)=\varnothing
$$

De modo similar, para sentenças contraditórias $\perp, \neg \perp$ inclui todos os mundos possíveis, o que, através da definição (1), gera o paradoxo de Bar-Hillel e Carnap (Floridi, 2004):

$$
\text { Cont }(\perp)=W
$$

A intuição geral por detrás do paradoxo é que a informatividade de uma sentença $s$ é inversamente proporcional à probabilidade dos estados de coisas que $s$ descreve se for o caso.

Bar-Hillel e Carnap (1953) também formularam uma maneira de mensurar o conteúdo. A mensuração $m$ é obtida através da distribuição de probabilidades a priori sobre o conjunto de todos os mundos possíveis, sendo que a soma dos valores distribuídos é 1. Desse modo, a teoria da informação semântica tradicional define o conteúdo (Cont) de uma sentença s como a mensuração do complemento de s:

- definição [2]: $\quad$ Cont $(s)=d f^{1-m(s)}$ 
Para uma sentença logicamente verdadeira $T$ temos:

$$
m(T)=1
$$

Uma sentença logicamente verdadeira (verdadeira em todos os mundos) retorna uma medida mínima de conteúdo, e a partir da definição [2] temos:

$$
\operatorname{Cont}(T)=1-1=0
$$

Uma sentença contraditória (inconsistente) $\perp$ é falsa em todos os mundos, portanto:

$$
m(\perp)=0
$$

Uma sentença contraditória vai retornar uma medida máxima de conteúdo. A partir da definição [2] e de $m(\perp)=0$, temos a segunda instância do paradoxo:

$$
\text { Cont }(\perp)=1-0=1
$$

Com respeito a sentenças inconsistentes Bar-Hillel e Carnap comentam (1953, p. 229):

It might perhaps, at first, seem strange that a self-contradictory sentence, hence one which no ideal receiver would accept, is regarded as carrying with it the most inclusive information. It should, however, be emphasized that semantic information is here not meant as implying truth. A false sentence which happens to say too much is thereby highly informative in our sense. Whether the information it carries is true or false, scientifically valuable or not, does not concern us. A self-contradictory sentence asserts too much; it is too informative to be true.

Foi para evitar uma teoria semântica da informação que tinha como um dos principais resultados "sentenças muito informativas para serem verdadeiras" que Floridi (2004) elaborou a Teoria da Informação Fortemente Semântica (TIFS).

\section{Lógica de estar informado}

Antes da defesa da TIFS é necessário passar em resumo como funciona a lógica da informação semântica de Floridi (2006), pois o seu exame em muito contribui para a compreensão sobre por que a TIFS não é arbi- 
trária, já que justifica a inclusão e supressão de alguns axiomas comuns a lógicas modais epistêmicas, entre eles o $A_{4}$, o axioma da veracidade.

Como qualquer um familiarizado com lógica modal sabe, a lógica epistêmica $(E L)$ formaliza a relação "a sabe que $p$ " $\left(K_{a} p\right)$, enquanto a lógica doxástica $(D L)$ formaliza a relação "a acredita que $p$ " $\left(B_{a} p\right)$. O que Floridi (2006) argumenta na formulação dessa nova lógica é que a $I L$ pode fazer por "estar informado" o que a $E L$ faz por "saber" e a $D L$ faz por "acreditar". Os que rejeitam que a lógica epistêmica consegue capturar formalmente a relação de conhecimento e que a lógica doxástica consegue capturar formalmente a relação de crença, certamente rejeitarão que a noção de estar informado poderá ser capturada combinando-se determinadas características de tais lógicas. Construir agentes epistêmicos racionais diretamente através da axiomatização de uma lógica é um procedimento que é justificado apelando-se para o significado de cada axioma (Lemmon e Scott, 1977). Dito de outra maneira, ache uma estória epistemológica plausível para justificar a semântica da lógica que se pretende elaborar. Para a prática filosófica, não adianta cálculo sem intuição. Criar novas lógicas é uma tarefa que nada acrescenta se não houver ao menos uma aplicação ou uma boa justificativa, no nosso caso ela é de cunho epistemológico. O que devemos evitar é cair numa espécie de "negligência epistêmica" (Chrisholm, 1963).

A proposta desenvolvida no artigo de Floridi (2006) é que a lógica modal normal (NML) KTB (também conhecida como B, Br ou o sistema de Brouwer ${ }^{2}$ ) é adequada para formalizar a relação de "estar informado" e, portanto, que a IL pode ser construída como uma leitura informacional de KTB. A semântica kripkiana (Kripke, 1963), a linguagem habitual da lógica clássica, do cálculo proposicional (CP) e da lógica modal normal proposicional (Girle, 2000) são pressupostas. Implicação $(\rightarrow)$ é usada no sentido "material"; as letras gregas são variáveis proposicionais metalinguísticas com alcance sobre fórmulas bem formadas da linguagem objeto da $N M L$ correspondente.

Segundo Floridi (2006, p. 435) "informação" pode ser compreendida de várias maneiras, por exemplo, como sinais, padrões naturais ou regularidades nômicas, como instruções, como conteúdo, como notícias, como sinônimo de dados, como poder ou recurso econômico e assim por diante. É controverso se todos ou ao menos a maior parte desses sentidos de "informação" podem ser reduzidos a um conceito fundamental, como mencionado na introdução ${ }^{3}$. O tipo de "informação" que interessa é a

2 Para uma descrição de KTB ver Hughes \& Cresswell (1996) e Chellas (1982, p. 131).

3 Floridi $(2004,2005)$ é cético sobre tentativas de achar uma teoria unificada da informação e, portanto, uma lógica única que capturaria todas as características interessantes. 
informação como conteúdo semântico que, por um lado, é concernente a um estado de um sistema, e que, por outro lado, permite a elaboração de um conhecimento proposicional de um agente sobre aquele estado do sistema. É o sentido no qual Matthew é informado que $p$, por exemplo, que "o trem para Londres sai às 10h30min da manhã", ou sobre o estado de coisas $f$ expresso por $p$, por exemplo, o quadro de horários da via férrea. É nesse sentido intuitivo de um conteúdo declarativo, objetivo e semântico que $p$ ou sobre $f$ que falaremos de "informação" daqui para frente. Esse sentido pode ser qualificado de modo licencioso como "cognitivo", um rótulo neutro útil para se referir aqui a toda uma família de relações que expressam atitudes proposicionais, incluindo "saber", "acreditar", "lembrar", "perceber", "experimentar" e "considerar". Qualquer sentido "não-cognitivo" de "informação semântica" será ignorado4.

O que Floridi fez foi o seguinte. Escolheu os três sistemas epistêmicos mais populares, a saber, os sistemas KT, S4 e S5 e os três sistemas doxásticos mais populares, a saber KD, KD4 e KD45 (Girle, 2000, p.148) e os dispensou, perguntando se havia alguma lógica modal normal que não pertencesse a esses sistemas que poderia formalizar corretamente "a está informado que $p$ ". Pois ao interpretarmos tais lógicas cognitivamente é claro que muitas vezes "estar informado" pode ser corretamente analisado como sinônimo de "saber" ou "crer". No entanto, não é o objetivo inicial proposto compreender como uma lógica da informação pode colapsar de forma trivial com alguma lógica epistêmica ou lógica doxástica, pelo contrário, o problema interessante é mostrar as propriedades que não são compartilhadas com tais lógicas conhecidas. Daí dispensar os sistemas mais populares, senão a lógica proposta nada mais seria do que uma lógica ou epistêmica ou doxástica, o que, nas palavras de Floridi, seria "cheap". De certo modo "espremido" entre os sistemas KT, KDB e S5 fica o pouco explorado KTB, ao qual Chellas, por exemplo, dedica uma página somente no seu importante livro Modal Logics (Chellas, 1982, p. 131), e que é interessante por compartilhar de alguns axiomas com tais sistemas vizinhos, mas não todos. Em breve, veremos como o sistema KTB-IL é montado. Por ora vejamos como é modelado "estar informado".

O mesmo procedimento padrão que adotamos na lógica epistêmica é adotado na lógica da informação. O operador modal de necessidade é interpretado como "está informado que", ou seja, substituímos o

4 Os exemplos que Floridi apresenta são os de uma estipulação ("seja o valor de $\mathrm{x}=3$ " ou "suponha que descobrimos os ossos de um unicórnio"), um convite ("você está cordialmente convidado à festa da faculdade"), uma ordem ("feche a janela!"), uma instrução ("para abrir a caixa vire a chave"), um movimento no jogo ("1.e2-e4 c7-c5" no começo de um jogo de xadrez). Todos podem ser corretamente qualificados como tipos de informação compreendidos como conteúdo semântico. 
"necessário" pela noção cognitiva que estamos interessados, mantendo as propriedades do operador e interdefinições usuais, como será exposto. Ao invés de $K$, claro, usamos o símbolo $I$ e fazemos referência explícita ao agente informado a. Traduzimos "a está informado que $p$ ":

$$
\square p=I_{a} p
$$

Definimos o operador de possibilidade $\rightarrow$ de modo usual e a ele atribuímos a leitura de desinformação (ou não-informado, não possui a informação) que não $p(\neg p)$ e usamos o símbolo $U$. Ou seja, por toda a informação que a possui é possível que $p$, o que traduzimos assim:

$$
U_{a} p={ }_{\text {def }} \neg I_{a} \neg p
$$

\begin{tabular}{|c|c|c|c|}
\hline Nome & Definições & $\begin{array}{l}\text { Axioma ou NML } \\
\text { correspondente }\end{array}$ & $\begin{array}{l}\text { Propriedade } \\
\text { da estrutura }\end{array}$ \\
\hline$A_{1}$ & $\varphi \rightarrow(x \rightarrow \varphi)$ & $1^{\circ}$ axioma do $\mathrm{CP}$ & \\
\hline$A_{2}$ & $(\varphi \rightarrow(x \rightarrow \psi)) \rightarrow((\varphi \rightarrow x) \rightarrow(\varphi \rightarrow \psi))$ & $2^{\circ}$ axioma do $\mathrm{CP}$ & \\
\hline $\mathrm{A}_{3}$ & $(\neg \varphi \rightarrow \neg x) \rightarrow(x \rightarrow \varphi)$ & $3^{\circ}$ axioma do $\mathrm{CP}$ & \\
\hline$A_{4}$ & $\square \varphi \rightarrow \varphi$ & K2, Veracidade & Reflexiva \\
\hline$A_{5}$ & $\square(\varphi \rightarrow x) \rightarrow(\square \varphi \rightarrow \square x)$ & K, Distribuição & Normal \\
\hline$A_{6}$ & $\square \varphi \rightarrow \square \square \varphi$ & KK, Introspecção Positiva & Transitiva \\
\hline$A_{7}$ & $\varphi \rightarrow \square \vee \varphi$ & KTB, Axioma de Brouwer & Simétrica \\
\hline$A_{8}$ & $\Delta \varphi \rightarrow \square \Delta \varphi$ & S5, Introspecção Negativa & Euclideana \\
\hline$A_{9}$ & $\square \varphi \rightarrow \Delta \varphi$ & KD, Consistência & Serial \\
\hline$A_{10}$ & $(\square(\varphi \rightarrow x) \rightarrow(\square(x \rightarrow \psi) \rightarrow \square(\varphi \rightarrow \psi)))$ & $\begin{array}{l}\text { Transmissão de um único } \\
\text { agente }\end{array}$ & \\
\hline$A_{11}$ & $\left(\square_{x} \square_{y} \varphi \rightarrow \square_{x} \varphi\right)$ & $\begin{array}{l}\text { K4, Transmissão de } \\
\text { múltiplos agentes }\end{array}$ & \\
\hline
\end{tabular}

Quanto aos axiomas foi adotada uma nomenclatura de $A_{1}$ até $A_{11}$ :

Reprodução e tradução da tabela com permissão do autor (Floridi, 2006, p. 441).

Os primeiros axiomas são os menos problemáticos, $A_{1}, A_{2}$ e $A_{3}$, pois se estamos falando de lógicas normais, os três primeiros devem ser assumidos trivialmente. Além do axioma $A_{5}$, que é válido para todas as estruturas possíveis das $N M L$, outros dois axiomas que são satisfeitos pela $I L$ são $A_{4}$ e $A_{9}$, respectivamente o da veracidade e o da consistência. Comecemos pelo menos problemático. Floridi cita quatro motivos (2006, p. 442) pelos quais devemos incluir $A_{9}$ no nosso esquema de axiomas. No entanto, esses quatro motivos na verdade são variações de um motivo que apenas define o que entendemos por "consistência". Se um 
agente a possui a informação que o seu avião decola às $15 \mathrm{~h}$. no dia $x$, então ele não possui a informação de que o avião não decola às 15h. no dia $x$. O ponto é simplesmente que agentes informacionais (que detém informações) não podem ser inconsistentes ao avaliar as possíveis informações que possuem, senão falhariam não apenas em possuí-las, mas também em transmiti-las. Agentes informacionais inconsistentes devem ser desconsiderados. Se estivéssemos versando sobre a lógica de "tornar-se informado", não seria problemático que aceitássemos que um agente pudesse receber e deter por um tempo duas mensagens ${ }^{5}$ contraditórias, por exemplo, você pode ler no quadro de avisos que o voo sai às 15h. e ouvir de um amigo que o mesmo voo não sai às15h. Como uma informação anula a outra por ser tratada como um caso de negação clássica, $p$ e $\neg p$. O que acontece é simplesmente que o agente torna-se incapaz de subjetivamente identificar qual informação ele detém, qual das duas mensagens é de fato informação, o que nos leva ao outro axioma ligado ao da consistência, o da veracidade, pois se satisfaz $A_{4}$, satisfaz a fortiori $A_{9}$.

$\mathrm{O}$ que significa dizer que, se a IL satisfaz a tese da veracidade, ela satisfaz a da consistência também, inexoravelmente? Para começar, não há informação verdadeira que não seja consistente, e isso não é problemático. $\mathrm{O}$ motivo principal de introduzir a consistência antes da veracidade é para mostrar que tais noções estão intimamente relacionadas, mas não apenas isso. No exemplo anterior em que o agente a recebe duas mensagens contraditórias, mesmo que esse se decida por acabar com a contraditoriedade e tomar partido de apenas uma mensagem, só poderá considerar-se informado ao descobrir qual das duas mensagens é de fato verdadeira e, ainda assim, se tiver optado pela mensagem falsa, saberá que de fato nunca esteve informado até o ponto no tempo em que descobre qual é a mensagem verdadeira, a informação. Nesse ponto, informação funciona de modo similar à noção de conhecimento. Ambos são necessariamente verdadeiros. Falsa informação é como um falso amigo, um falso amigo ou é inimigo ou uma pessoa dissimulada e que também não se qualifica como portador de amizade. Claro que a mensagem falsa ainda possui conteúdo semântico, no entanto não satisfaz $A_{4}$. Parece que isso também não é problemático, no entanto não é o que pensa Crnkovic (2005).

Até agora, a lógica da informação não trouxe nenhuma diferença fundamental para não colapsar na lógica epistêmica, que também satisfaz os axiomas examinados. Seria a IL uma mera EL? A análise dos próximos axiomas mostra que não. Os axiomas que fazem a diferença

\footnotetext{
5 Percebam que aqui não é usado o termo "informação".
} 
entre a lógica da informação e seus primos epistêmico e doxástico são os $A_{6}$ e $A_{8}$, a lembrar, introspecção positiva e introspecção negativa, não contemplados na $I L$ :

$$
\begin{array}{ll}
A_{6} & \square \varphi \rightarrow \square \square \varphi \\
A_{8} & \diamond \varphi \rightarrow \square \vee \varphi
\end{array}
$$

Em que o agente informacional teria o equivalente na lógica epistêmica ao que Hintikka chamou das teses $K K$ (Hintikka, 1962, p. 16) e $B B$, ou seja, quando um agente sabe que sabe e quando acredita que acredita. "Crer" ou "acreditar" e "conhecer" são estados cognitivos capazes de implementar uma relação de "acesso privilegiado", chamada por Williamson (2002) de transparência ${ }^{6}$, o que tornaria agentes possuidores de tais estados mentais "agentes cartesianos", ou seja, o que quer que seja que torna possível um agente a acreditar (ou saber) que $p$, isso também é o que torna possível para a acreditar (ou saber) que a acredita (ou sabe) que $p$. Trocando em miúdos, defensores de $A_{6}$, e no caso da informação este axioma não é contemplado, alegam que se um agente a acredita (ou sabe) que $p$, esse é um fato mental interno que também pode ser, em princípio, mentalmente acessível ao agente cartesiano. Esse agente seria, portanto, capaz de adquirir um estado mental reflexivo, seja de acreditar que acredita que $p$, seja de saber que sabe que $p$.

Floridi traduz esse estado mental reflexivo para linguagem da Teoria da Comunicação (Shannon e Weaver, 1949) e diz que, se há um canal de comunicação que permite que a tenha acesso à sua condição doxástica ou epistêmica, esse deve ser um canal único (single-channel), que não requer um segundo canal, um meta-canal de comunicação. Isso não acontece no caso de "estar informado". Deter uma informação não requer necessariamente que exista um ato mental consciente que visa o estado de estar informado. O mote de Floridi é que crença e conhecimento estão na cabeça, mas informação pode estar no bolso (ou no HD). Dito de outro modo, agentes, sejam eles biológicos ou artificiais, podem deter a informação que $p$, sem que possuam mentes ou algum tipo de estado mental que concerne $p$. "Estar informado" não deve ser analisado como provendo um acesso privilegiado a $p$, ou seja, "estar informado" não é necessariamente portadora de transparência como em "conhecer" e "acreditar". Porém, a preocupação central do presente artigo é a tese da veracidade, então é melhor focar no objetivo proposto e tentar defendê-lo.

6 Timothy Williamson argumenta contra $K K$ e $B B$ (2000) e contra $K_{a} \rightarrow B_{a}$ (2002). "Transparente" devido à sua natureza introspectiva de acesso direto e privilegiado, contrapõe-se a "Opaca" devido à sua natureza indireta, não refletiva ou inconsciente.

7 Agentes cartesianos simplesmente porque sabem que sabem. 


\section{Informação e verdade}

Assim como Crnkovic (2005), também Fetzer (2004), Devlin (1991) e Scarantino e Piccinini (2010) criticam a noção de que a informação deve ser verdadeira sempre. Para tais autores, dados bem-formados e com significado já poderiam ser qualificados como informação, não importa se representam ou transmitem uma verdade ou falsidade ou não tenham sequer valor alético.

Porém alguma sedimentação é necessária nesse ponto. Entendemos que dados bem-formados e com significado são o conteúdo semântico. Padrões de dados frequentemente constituem sentenças na linguagem natural, mas é claro que também podem vir a constituir mapas, fórmulas, vídeos, diagramas ou quaisquer outras construções semióticas sobre uma variedade de codificações físicas, sendo determinadas pela sintaxe apropriada (boa-formação) e pela semântica (significado). Para o nosso propósito epistêmico, devemos destacar que o conteúdo semântico em questão trabalha como uma interface entre um sistema $A$ e um agente a, por exemplo:

a) um sistema $A$ em um estado específico, digamos, Brasília e o seu papel como capital do Brasil, o que pode ser capturado através de diferentes análises de dados (descrever, modelar, representar, etc., $A$ ).

b) um agente a, que pode vir a conhecer $A$ através de diversas elaborações dos dados relevantes (aquisição, interpretação, compreensão, etc., dos dados).

Esse sentido epistêmico de "informação" pode ser reconhecido de modo imediato, quando traduzido em atitudes proposicionais tais como "João está informado (detém a informação) que Brasília é a capital do Brasil".

Floridi $(2004,2005,2006,2007)$ argumenta que a definição de informação em termos de conteúdo aleticamente neutro - padrões de dados bem-formados que podem ser qualificados adicionalmente como verdadeiros ou falsos - fornece condições necessárias, porém insuficientes. Se $p$ deve contar como informação, $p$ deve ser verdadeiro, e isso nos leva à definição (DEF) de informação fortemente semântica de Floridi:

(DEF) $p$ é informação se e somente se $p$ é constituída por dados bemformados, com significado e verdadeiros.

A tese da veracidade incorporada na definição corresponde à caracterização da definição de "conhecimento". Formalizamos "a está 
informado que $p$ " como $I_{a} p$, então a tese da veracidade da informação semântica formulada através do axioma $A_{4}$ temos ${ }^{8}$ :

- Tese da Veracidade: $I_{a} p \rightarrow p$

E interpretamos: a está informado que $p$ somente se $p$ é verdadeiro.

O problema é que os oponentes da definição (DEF) argumentam que, quando analisamos o conteúdo da informação semântica, factual e orientada epistemicamente, uma lógica da informação que satisfaz a tese da veracidade pode ser inadequada por ser muito forte.

Vamos ver como Foridi defende a tese da veracidade, para que possamos mostrar como a sua defesa captura intuições modais importantes sobre a informatividade. O vocabulário a seguir será usado (Floridi, 2007, p. 34):

$D=\left\{p_{1}, \ldots p_{n}\right\} ; D$ é um (possivelmente vazio) domínio de proposições;

$\varphi, \psi=$ variáveis proposicionais com alcance sobre $D$;

$S=\left\{i_{1}, \ldots i_{n}\right\} ; S$ é um (possivelmente vazio) domínio de instâncias da informação;

$t(\varphi)=\varphi$ é contingentemente verdadeiro;

$f(\varphi)=\varphi$ é contingentemente falso;

$t / f(\varphi)=\varphi$ é contingentemente verdadeiro ou falso;

$T(\varphi)=\varphi$ é uma tautologia;

$C(\varphi)=\varphi$ é uma contradição;

$H(\varphi)=$ conteúdo informativo primário (não derivado) de $\varphi$;

$P(x)=$ probabilidade de $x$.

O argumento semântico é baseado em quatro princípios, independentemente de como os membros de $S$ são definidos:

P1. $\forall x H(x) \geq 0$;

Princípio da Natureza não-negativa da Informação: diz que nenhuma instância da informação pode ter um conteúdo informativo primário negativo.

P2. $\forall x \forall y((x \neq y) \supset(H(x \cup y)=H(x)+H(y)))$;

Princípio Aditivo: diz que para quaisquer duas diferentes instâncias da informação, o seu conteúdo informativo geral é igual à soma dos seus conteúdos informativos (ou informacionais).

P3. $\forall \varphi(\mathrm{P}(\varphi)=1) \supset(H(\varphi)=0))$;

Princípio da Relação Inversa: diz que qualquer proposição que tem probabilidade 1 não possui conteúdo informativo.

P4. $\forall \varphi(H(\varphi)=0) \supset \neg(\varphi \in S))$;

Complementa P3 e diz que qualquer proposição sem conteúdo informativo não pode ser qualificada como informação.

8 Um dos problemas em aberto na filosofia da informação é se há uma lógica da informação $(I L)$, diferente da lógica epistêmica $(E L)$ e da doxástica $(D L)$, que formaliza a relação "a está informado que $p^{\prime \prime}\left(I_{a} p\right)$ satisfatoriamente. 
P1 e P2 dizem respeito a $S$ e da natureza cumulativa de conteúdos informacionais. P3 e P4 dizem respeito a $D$ e à relação entre informação e probabilidade.

A estratégia para inocular a tese da veracidade se dá da seguinte maneira. Primeiro é assumido que os opositores da tese estão corretos. Depois veremos como o que eles defendem é permissivo em demasia, ao passo que, ao restringirmos as consequências indesejadas veremos que a definição de informação a que chegamos já contempla a tese da veracidade intrinsecamente.

O primeiro passo consiste em aceitar (temporariamente) a posição segundo a qual, ao se equacionar $S$ para $D$, ou seja, o domínio das instâncias da informação para o domínio das proposições, tomaremos que todas as proposições, independentemente dos respectivos valores de verdade, contam como instâncias de informação.

Como consequência de P3 e P4 temos:

i) $\quad T(\varphi) \supset(P(\varphi)=1)$;

ii) $(P(\varphi)=1) \supset \neg(\varphi \in S)$; portanto,

iii) $T(\varphi) \supset \neg(\varphi \in S)$.

Tautologias não são instâncias da informação. Ninguém pode informar um outrem sobre o resultado específico de um jogo de "cara ou coroa" dizendo que "vai dar cara ou coroa", pois isso já é sabido de antemão. Tautologias são casos-limite, pois representam instâncias de informação desprovidas de qualquer informatividade. Portanto, é nesse sentido que aceitarmos que $D=S$ é permissivo demais e que deve ser revisto.

\section{Restrições e requerimentos}

Na seção II mostramos como se dá o paradoxo de Bar-Hillel e Carnap (1953) ilustrando como conteúdo e intensão se articulam na semântica de Carnap. Vejamos agora como Floridi (2007) exibe uma instância do paradoxo, ao tentar restringir aleticamente a extensão do conceito de informação para evitar o problema com as tautologias. Ao acrescentar a seguinte restrição:

$$
\forall \varphi((T(\varphi) \supset(H(\varphi)=0)) \supset \neg(\varphi \in S))
$$

[fórmula 1]

É indicado (através de P1 - P4) que infelizmente ainda ficamos com o seguinte resultado:

i) $(P(\varphi)<1) \supset(\varphi \in S)$;

ii) $C(\varphi) \supset(P(\varphi)=0)$; portanto,

iii) $C(\varphi) \supset(\varphi \in S)$. 
Este é o paradoxo de Bar-Hillel e Carnap formulado de modo sucinto, de acordo com o qual uma sentença contraditória carrega o máximo de informação (imaginem o caso de perguntar para um amigo que está em um local específico $l$, em um tempo $t^{9}$, se está chovendo, ao passo que ele responde "chove e não chove"). Contradições têm probabilidade zero, mas são muito informativas, as mais informativas das proposições. Dito de outra maneira, a mais improvável das mensagens, uma contradição, carregaria o máximo de informação que um agente pode receber.

Se antes tentamos restringir aleticamente a extensão do conceito de informação para evitar o problema com as tautologias, parece que agora temos que tentar excluir também as contradições.

É tentador restringir o caso das contradições através da própria condição de veracidade, ou seja, que somente sentenças verdadeiras contam como informação, e por isso contradições não contariam como informação. Só que isso não é uma defesa da tese da veracidade, é uma petição de princípio. Um opositor à tese da veracidade pode acrescentar que só devemos incluir no conjunto original de proposições aquelas que são contingentemente verdadeiras ou contingentemente falsas. E depois o opositor argumentaria que somente esses tipos de proposições poderiam ser qualificadas como informação, independentemente dos valores de verdade contingentes. A nova restrição ficaria assim:

$$
\forall \varphi((\mathrm{T}(\varphi) \vee \mathrm{C}(\varphi)) \supset \neg(\varphi \in \mathrm{S}))
$$

[fórmula 2]

Que modela a informação do mesmo modo que opositores à tese da veracidade como Crnkovic (2005). Opositores que aceitam tal resultado acreditam que proposições tautológicas e contraditórias não podem ser qualificadas como informação, porque são não informativas por princípio, o que é um resultado bem aceito por todos os teóricos da informação, mas ainda assim argumentam que proposições contingentemente falsas devem contar como informação porque podem ser úteis informativamente (de modo heurístico) ou contrafactualmente informativas a respeito de como poderia ser o caso.

Por mais intuitivo que isso possa parecer, tal posição é insustentável, porque exclui a possibilidade da informação ser eliminada (apagada ${ }^{10}$ ) sintaticamente através da geração de inconsistências, ou seja, o mesmo $\varphi$ é afirmado e negado de modo independente do seu valor de verdade ou interpretação semântica. Se considerarmos P1 - P4 e a fórmula [2], temos que (Floridi, 2007, p. 37):

9 A especificidade é importante para mantermos a consistência.

10 Uma das operações do conjunto $D$ é a de erase, além de write e read. 


$$
\forall \varphi \forall \psi((\varphi \neq \psi \wedge t / f(\varphi) \wedge t / f(\psi)) \supset(0<H(\varphi)<H(\varphi \cup \psi)>H(\psi)>0)) \quad \text { [fórmula 3] }
$$

A fórmula acima afirma que, se temos duas diferentes proposições contingentes, então a união de seus conteúdos informacionais é sempre maior do que o conteúdo informativo de cada proposição considerada separadamente. Infelizmente, a fórmula [3] tem um resultado altamente contraintuitivo (curiosamente, a partir de uma posição aparentemente intuitiva), a saber, que ao acumularmos quaisquer proposições contingentes sempre estaremos aumentando o nosso estoque de informações, independentemente do caso das proposições serem mutuamente inconsistentes. Gerando um depósito de informações contraditórias, o que endossa a fórmula implausível:

$$
H\left(\cup_{1}^{\mathrm{n}} \varphi\right)<H\left(\cup_{1}^{\mathrm{n}+1} \varphi\right)
$$

[fórmula 4]

De modo sucinto, quanto mais proposições contingentes forem acumuladas, por exemplo, somadas a um banco de dados, mais informativo torna-se o banco de dados, o que é obviamente falso. Isso é óbvio, porque se temos proposições que negam outras proposições no mesmo banco de dados ambas se anulam e não há acréscimo algum de informação.

Uma maneira de tentar atenuar o problema com a fórmula [4] é adicionar a cláusula de que $\varphi$ pode ter alcance sobre proposições informacionalmente equivalentes, tais como "João dirige o carro" e "o carro é dirigido por João", além de tautologias, o que fazemos simplesmente substituindo o sinal de $<$ por $\leq$ :

$$
H\left(\cup_{1}^{\mathrm{n}} \varphi\right) \leq H\left(\cup_{1}^{\mathrm{n}+1} \varphi\right)
$$

[fórmula 5]

Porém, mesmo segundo a fórmula [5] não há possibilidade de decréscimo dos conteúdos informacionais a não ser que os dados sejam danificados ou apagados fisicamente. Isso é bom demais para ser verdade. Imaginem um mundo no qual, através do acúmulo aleatório de proposições contingentes, ainda sempre aumentássemos o nosso repositório de informações. Esse é o mundo da fórmula [5].

Um parâmetro fundamental que deve ser respeitado é o da consistência. Veremos a seguir como eliminar a inconsistência do nosso modelo de informação.

Floridi (2007) sugere que devemos respeitar dois requerimentos para assegurarmos que a análise do que vai contar como informação não se torne inflacionada.

Requerimento [1]: conteúdos informacionais podem decrescer sintaticamente sem que sejam apagados ou danificados fisicamente. 
Simbolicamente:

$$
\diamond\left(H\left(\cup_{1}^{\mathrm{n}} \varphi\right)>H\left(\cup_{1}^{\mathrm{n}+1} \varphi\right)\right.
$$

[fórmula 6]

É necessário fazer um adendo. Floridi não quer dizer que não há trabalho físico, por exemplo, quando é apagada alguma informação em um disco rígido (HD). O requerimento [1] indica somente que através de operações sintáticas deve ser possível que haja decréscimo de conteúdo, ou seja, que um estado anterior ao do acréscimo de uma proposição seja mais informativo que o estado posterior $(n+1)$. O que deve ficar claro que é através de uma regra sintática que isso deve ser disparado.

Requerimento [2]: É improvável que um repositório de informações aumente, ao adicionarmos qualquer proposição contingente.

O requerimento [2] tem a função de qualificar o requerimento [1] de modo entrópico. Diz que quanto maior o repositório $D$, ao adicionarmos qualquer $\varphi$ contingente ao repositório, cai a probabilidade de que o conteúdo informativo de $D$ aumente, ou pelo menos a probabilidade é a mesma do que era antes da adição.

Simbolicamente:

$$
\begin{array}{ll}
P\left(H\left(\cup_{1}{ }^{\mathrm{n}} \varphi\right) \geq H\left(\cup_{1}{ }^{\mathrm{n}+1} \varphi\right)>P\left(H\left(\cup_{1}^{\mathrm{n}} \varphi\right)<H\left(\cup_{1}{ }^{\mathrm{n}+1} \varphi\right)\right.\right. & \text { [fórmula 7] } \\
P\left(H\left(\cup_{1}{ }^{\mathrm{n}} \varphi\right) \geq H\left(\cup_{1}{ }^{\mathrm{n}+1} \varphi\right)=P\left(H\left(\cup_{1}{ }^{\mathrm{n}} \varphi\right)<H\left(\cup_{1}{ }^{\mathrm{n}+1} \varphi\right)\right.\right. & \text { [fórmula 8] }
\end{array}
$$

Notemos que a fórmula sete é mais forte que a fórmula oito, pois na última ainda há a possibilidade de que a probabilidade se mantenha a mesma após o acréscimo de uma proposição contingente qualquer.

Tanto o requerimento [1], que é traduzido através da fórmula [6], quanto o requerimento [2], que tem as suas versões nas fórmulas [7] e [8], precisam de mais um passo para que tautologias e contradições não sejam qualificadas como informação. As restrições reformuladas para dar conta de tautologias e contradições são, simbolicamente:

$$
\begin{gathered}
\forall \varphi((T(\varphi) \vee C(\varphi)) \supset \neg(\varphi \in S)) \neg C\left(\cap_{1}{ }^{x} \varphi\right) \\
\diamond\left(H\left(\varphi^{\mathrm{n}} \varphi\right) \leq H\left(\cup_{1}{ }^{\mathrm{n}+1} \varphi\right)\right.
\end{gathered}
$$

[fórmula 9]

Duas consequências imediatas de [9] são, primeiramente, que agora é permitido que o conteúdo informacional decresça sintaticamente e, em segundo lugar, se esse conteúdo aumentar será uma tarefa bem mais trabalhosa graças ao tratamento dos casos de tautologia e contradição devidamente controlados. 
A penúltima manobra consiste em mostrar que, apesar de termos, até agora, como dar conta do problema sintático, o modelo de informação implementado por [9] só satisfaz os requerimentos [1] e [2] de modo parcial, pois o conteúdo informacional também deve poder decrescer semanticamente.

Um exemplo simples e que ilustra bem o problema é o caso de um livro (blocos de notas, arquivos de texto em computadores, celulares e afins também são bem-vindos para fins de exemplificação). Vamos, para fins de economia, tomar o exemplo de um ebook - A Era dos Extremos, de Eric Hobsbawn. (a) As informações no ebook podem ser perdidas, se o HD do computador queimar (e você não tiver backup, claro) - perda física da informação. (b) As informações do ebook podem ser perdidas por causa de um vírus ou arquivo malicioso que pode reprogramar todo o texto de modo a embaralhar todas as letras (perda sintática da informação) ou (c) algo diferente pode também acontecer. Algum brincalhão (e não é o brincalhão a presente preocupação) pode modificar com algum editor de pdf qualquer parte do texto, e onde líamos "Em Agosto de 1945 os Estados Unidos da América jogaram bombas sobre Hiroshima e Nagasaki" agora lemos "Em Agosto de 1945 os Estados Unidos da América jogaram flores sobre Hiroshima e Nagasaki".

De acordo com [9], não há perda no caso (c), se analisamos a modificação de modo exclusivamente sintático, quantitativo. Mas, é um evidente absurdo quando analisamos semanticamente. A primeira sentença conta como informação, pois os Estados Unidos de fato jogaram bombas em Hiroshima e Nagasaki em agosto de 1945. A segunda sentença não conta como informação, pois é falsa. Um terceiro requerimento é então introduzido:

Requerimento [3] - O conteúdo informativo pode ser perdido tanto fisicamente, quanto sintaticamente e semanticamente.

A perda de informação pode acontecer (a) através de negação, (b) através de falsificação, (c) tornando-as satisfeitas em todos os mundos possíveis (caso limite representado pelas tautologias) ou (b) tornando as proposições inconsistentes.

Simbolicamente:

$\diamond\left(H\left(\cup_{1}{ }^{\mathrm{n}} \varphi\right)>H\left(\cup_{1}{ }^{\mathrm{n}+1} \varphi\right)\right.$ fisicamente, sintaticamente e semanticamente.

Para finalizar, é acrescentado o que chamamos de intuição modal importante no início deste trabalho, motivado pela Restrição [3] + fórmula [10] - somente proposições contingentemente verdadeiras contam como informação.

$$
\forall \varphi((\varphi \in S) \supset t(\varphi))
$$


O que não é nada mais do que a Tese da Veracidade reformulada ${ }^{11}$, que mostra não apenas que [DEF] está correta, mas também que o conteúdo informacional pode decrescer, crescer, além de poder ser perdido fisicamente, sintaticamente e semanticamente. Também podemos ilustrar que apenas proposições contingentemente verdadeiras contam como informação, se falarmos de mundos possíveis, da seguinte maneira:

Uma sentença declarativa $s$ é informativa $\leftrightarrow s$ individuar ao menos alguns, mas não todos $w_{i}$ a partir de $W\left(\right.$ em que $w_{i} \in W$ ).

\section{Conclusão}

A tese da veracidade exibida através da fórmula [11], que atesta que somente proposições contingentemente verdadeiras contam como informação, é fruto da restrição [3]. Apesar de ela conter a intuição modal importante do requerimento contingencial da informatividade, ela não deixa de ser uma noção idealizada.

Como noção idealizada, tal requerimento de que, para contar como informação, uma proposição deve ser contingentemente verdadeira, a tese da veracidade também apresenta como uma de suas motivações centrais a idealização dos nossos agentes e a investigação de mecanismos subjacentes, o que Stalnaker (1991) chama de motivação científica ${ }^{12}$.

Agentes realistas costumam tomar sentenças que selecionam todos os mundos possíveis como sendo informativas. Mas, se falamos sobre a sobrecarga de informação, por exemplo, ao não conseguimos dar conta do problema do fechamento dedutivo que está em todas as lógicas modais normais, é porque não somos logicamente oniscientes e é justamente sobre a falha da onisciência lógica que a teoria da veracidade idealiza.

Porém, a despeito da idealização, o requerimento de apenas proposições contingentemente verdadeiras contarem como sendo informação semântica permanece uma intuição modal importante. Pois, para uma sentença declarativa $s$ ser informativa (e a proposição ou as proposições por ela expressa(s)), em um sentido de "estar de posse de"

11 A tese da veracidade $\left(I_{a} p \rightarrow p\right)$, (Floridi, 2006).

12 Em um artigo sobre o problema da onisciência lógica, advindo da inclusão do axioma da distributividade A5 em todas as lógicas modais normais. A discussão é se há alguma razão positiva para a idealização dos nossos agentes na lógica epistêmica. Segundo Stalnaker (1991), uma das motivações principais é a investigação de mecanismos subjacentes, isto é, a interação de diversos componentes diferentes que compõem sistemas complexos talvez seja mais bem compreendida de modo isolado, mesmo que tais componentes não sejam encontrados isoladamente em um contexto realista. Ele chama a motivação para tal idealização de "científica", pois em ciência estamos acostumados a isso, a saber, isolar certos componentes de fatores externos que afetariam de modo indesejado o comportamento de determinados experimentos. 
e de certo modo útil do termo "informativa" (o sentido de um agente a deter a informação que $p$ ), ela deve demarcar qual o mundo, dentro do espaço modal, é de fato o mundo atual.

É um sentido de informatividade que tem como apelo a tentativa de localizar o nosso mundo dentre o espaço de mundos possíveis, de modo que tautologias e inconsistências fiquem fora do alcance. Não importa frente a quantas tautologias ou contradições estamos expostos, se estamos perdidos no espaço modal, a informação, através da leitura oferecida, oferece boas pistas de como podemos voltar para casa, o mundo atual.

\section{Referências}

BAR-HILLEL, Y.; CARNAP, R. "An Outline of a Theory of Semantic Information". In: BAR-HILLEL, Y. Language and Information. Selected Essays on Their Theory and Application. Reading-London: Addison-Wesley, 1964, p. 221-274.

BAR-HILLEL, Y. Language and Information. Selected Essays on Their Theory and Application. Reading-London: Addison-Wesley, 1964.

CARNAP, R. Meaning and Necessity. Chicago: University of Chicago Press, 1947.

CHELLAS, B. F. Modal Logic: An Introduction. Melbourne: Cambridge University Press, 1980.

CHISHOLM, R. "Contrary-to-Duty Imperatives and Deontic Logic". In: Analysis, 24 (1963), p. 33-36.

CMKOVIC, G. "System Modeling and Information semantics". In: BUBENKO, J.; ERIKSSON, O.; FERNLUND, H.; LIND, M. (eds.). Proceedings of the Fifth Promote IT Conference. Lund: Studentlitteratur, 2005.

DEVLIN, K. Logic and Information. Cambridge: Cambridge University Press, 1991.

FETZER, J. H. "Information, Misinformation, and Disinformation". In: Minds and Machines, 4 (2004): 2, p. 223-229.

FLORIDI, L. "In Defence of the Veridical Nature of Semantic Information". In: The European Journal of Analytic Philosophy, 3 (2007): 1, p. 1-18.

. "Outline of a Theory of Strongly Semantic Information". In: Minds and Machines, 14 (2004):2, p. 197-222.

. "Semantic Conceptions of Information". In: Stanford Encyclopedia of Philosophy: <http://plato.stanford.edu/entries/information-semantic/>, 2005.

. "Semantic Information and the Correctness Theory of Truth". In: Erkenntnis,

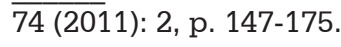

. "The Logic of Being Informed". In: Logique et Analyse, 49 (2006), p. 433-460.

FUHRMANN, A. "Reflective Modalities and Theory Change". In: Synthese, 81 (1989): 1, p. 115-134.

GIRLE, R. Modal Logics and Philosophy. London: McGill-Queen's University Press, 2000. 
B. G. Alonso - A tese da veracidade na teoria da informação ...

HINTIKKA, J. Knowledge and Belief. Cidade: Cornell University Press, 1962.

HUGHES, G. E.; CRESSWELL, M. J. A New Introduction to Modal Logic. New York: Routledge, 1996.

KRIPKE, S. "Semantical Analysis of Modal Logic I. Normal Propositional Calculi". In: Zeitschrift fur mathematische Logik und Grundlagen der Mathematik, 9 (1963), p. 67-96.

LEMMON, J.; Scott, D. An Introduction to Modal Logic. Oxford: Blackwell, 1977.

SCARANTINO, A.; PICCININI, G. "Information Without Truth". In: Metaphilosophy, 41 (2010): 3, p. 313-330.

SHANNON, C. E. Collected Papers. Los Alamos (California): IEEE Computer Society Press, 1993.

SHANNON, C. E.; WEAVER, W. The Mathematical Theory of Communication. [s.l.]: University of Illinois Press, 1949.

STALNAKER, R. "The Problem of Logical Omniscience". In: Synthese, 89 (1991): 3, p. 425-440.

Recebido em 29/01/2012.

Aprovado para publicação em 28/07/2012. 